\title{
Feasibility of a daily noise monitoring intervention for prevention of noise-induced hearing loss
}

\author{
Peter Rabinowitz, ${ }^{1}$ Deron Galusha, ${ }^{2}$ Linda F Cantley, ${ }^{2}$ Christine Dixon-Ernst, ${ }^{3}$ \\ Richard Neitzel (D) 4
}

'Department of Environmental and Occupational Health

Sciences, University of Washington, Seattle,

Washington, USA

${ }^{2}$ Occupational and

Environmental Medicine, Yale School of Medicine, New Haven, Connecticut, USA

${ }^{3}$ Otogienex, LLC, Pittsburgh, Pennsylvania, USA

${ }^{4}$ Department of Environmental Health Sciences, University of Michigan, Ann Arbor, Michigan, USA

\section{Correspondence to} Dr Peter Rabinowitz, Department of Environmental and Occupational Health Sciences, University of Washington, Seattle, Washington, USA; peterr7@uw.edu

Received 14 January 202 Accepted 3 June 2021

Published Online First 2 July 2021

\section{ABSTRACT \\ Background Despite the existence of hearing conservation programmes complying with regulatory standards, noise-induced hearing loss ( $\mathrm{NHHL}$ ) remains one of the most prevalent occupational diseases. Compulsory daily monitoring of noise exposure has been associated with decreased NIHL risk. We report on the experience of a voluntary daily noise monitoring intervention among noise-exposed workers.}

Methods Workers at three locations of a metals manufacturing company voluntarily used an in-ear noise monitoring device that could record and download, on a daily basis, the noise exposure inside of their hearing protection. We compared the hearing loss rates (in decibels hearing level/year) in these volunteers to controls from the same company matched for job title, age, gender, race, plant location, and baseline hearing level.

Results Over the follow-up period, 110 volunteers for whom controls could be identified monitored daily noise exposures an average of 150 times per year. Noise exposures inside of hearing protection were lower than ambient noise levels estimated from company records. While there was no significant difference in hearing loss rates between volunteers and controls, volunteers downloading exposures 150 times per year or had less hearing loss than those who downloaded less frequently. Conclusion These results indicate that voluntary daily noise exposure monitoring by workers is feasible and that greater frequency of downloading is associated with less hearing loss. If further development of noise monitoring technology can improve usability and address barriers to daily use, regular self-monitoring of noise exposure could improve the effectiveness of hearing conservation programmes.

Trial registration number NCT01714375.

\section{BACKGROUND}

Hearing loss is a chronic condition that not only leads to diminished health and productivity on its own, but is also associated with numerous other detrimental sequelae. ${ }^{12}$ Noise exposure in occupational settings is a major cause of hearing loss. ${ }^{3}$ According to national surveillance data from the US National Institute of Occupational Safety and Health, nearly $20 \%$ of workers in the manufacturing sector exhibit noise-induced hearing loss (NIHL). ${ }^{4}$ These workers may suffer increased depression, reduced social activity and potentially increased risk of cardiovascular disease ${ }^{25}$ and injuries. ${ }^{16}$

\section{Key messages}

What is already known about this subject?

- While it is known that high workplace noise levels lead to occupational hearing loss, and that mandatory monitoring can reduce risk, less is known about the feasibility of having workers voluntarily monitor their daily noise exposures.

\section{What are the new findings?}

- We found that volunteers in our study were able to record and download daily in-ear (inside of hearing protection) noise exposure data, with a mean of approximately 150 annual downloads.

- The noise levels inside of hearing protection were lower than the company estimates for ambient noise exposure. Volunteers who downloaded more than 150 times per year had less hearing loss than those volunteers who downloaded their exposures less frequently.

How might this impact on policy or clinical practice in the foreseeable future?

- Our findings support the feasibility of voluntary noise exposure monitoring.

- While it may currently be less effective than mandatory monitoring, the continued development of methods and procedures for workers to monitor personal noise exposures on a regular basis- future advancements in monitoring technology could lead to further reductions in risk of noise-induced hearing loss.

As a result of these adverse consequences of occupational noise exposure, mandatory hearing conservation programmes are required by the US Occupational Safety and Health Administration (OSHA) in settings where 8-hour time-weighted average (TWA) noise levels equal or exceed 85 dBA. Components of an OSHA-mandated hearing conservation programme include worker training, noise exposure assessment and control, routine audiometric testing, and the use of hearing protection devices (HPDs). Despite the specific requirement for each of these programme components, hearing loss prevention in many occupational settings relies largely on individual worker's use of HPDs. ${ }^{7}$ Evidence suggests that HPDs can decrease noise exposure significantly when used correctly, although there is substantial variability between 
types and brands of devices, ${ }^{7}$ and even among workers using the same devices. Several factors can influence workers' HPD use patterns. ${ }^{8}$ Qualitative data have demonstrated that workers' perception of noise, interest in hearing preservation and workplace interactions with others can influence HPD use among workers. ${ }^{9}$ Several interventions have been shown to improve HPD use among noise-exposed individuals. In particular, classroom instruction, distribution of HPDs, mailings and personal noise level assessments have all been shown to improve hearing protection use compared with audiometric testing alone. ${ }^{10}$

The Stages of Change model of behaviour change suggests that individuals progress through distinct phases during a transition towards healthy behaviour. This model has been used to evaluate the processes by which individuals access treatment after diagnosis with hearing loss. ${ }^{112}$ These studies have identified the stages of precontemplation, contemplation, preparation and action as the major phases for help seeking after diagnosis. ${ }^{11}$ Nearly $50 \%$ of the participants in these studies, even after diagnosis with hearing loss, were in the precontemplation and contemplation phases. Effective transitions from precontemplation to subsequent stages often require the delivery of information on health risks and exposures, ${ }^{13}$ and this model may be applicable to noise-exposed workers who have not yet been diagnosed with hearing loss. Individual-level information provision could potentially lead to more desirable HPD use behaviours and, therefore, decreased hearing loss over time. An improvement in hearing protection behaviour as a result of individual-level noise information provision has been demonstrated among construction workers, ${ }^{14}$ and mandatory use of in-ear noise dosimeters performing daily monitoring of noise inside of hearing protection has been associated with reductions in rates of hearing loss. ${ }^{15}$ However, whether daily noise exposure monitoring technology provides a benefit for the prevention of hearing loss among voluntary users has not been previously assessed.

In this study, we performed a longitudinal analysis of voluntary use of daily noise monitoring devices among noise-exposed industrial workers in order to evaluate the feasibility of such a voluntary approach and determine if workers who received information provided by individual in-ear noise dosimeters had decreased hearing loss compared with workers who did not receive information from dosimeters. We hypothesised that the information provided by the in-ear dosimeters would increase risk awareness and self-efficacy, allowing for a transition toward improved behaviours to prevent NIHL and, subsequently, reduced noise exposures.

\section{METHODS}

\section{Study design}

This study was conducted as a voluntary longitudinal trial of personal in-ear noise dosimeters among metals manufacturing plant workers in two locations: a complex of aluminium smelting facilities and a light metals fabrication facility. These facilities were part of an ongoing research collaboration between the study company and the Yale University School of Medicine, a collaboration that has been described in detail previously. ${ }^{16}$ The study was also registered on clinicaltrials.gov.

\section{Recruitment and retention}

We recruited noise-exposed volunteers on a rolling basis between 2008 and 2014 by distributing informational materials and holding meetings with groups of workers employed in operations that were included in the company's hearing conservation programme. The study team attempted to offer participation in the study to all noise-exposed workers in the study facilities. Company management was aware of the study but was not directly involved in subject recruitment, which was conducted by research staff. To be eligible for the study, volunteers had to be enrolled in their plant HCP and be over the age of 18 . After providing written informed consent, volunteers were fitted with the in-ear noise dosimeter and instructed regarding dosimeter use and exposure data downloading. Volunteers received points for each daily download of data; accumulated points were redeemable for gift cards. Participants were encouraged but not required to download their exposures on a daily basis. In addition, study volunteers received a gift card for completing baseline and annual questionnaires, as described later, as well as a small annual cash stipend. We report on a 5 -year follow-up with these volunteers.

\section{Daily noise exposure monitoring}

Volunteers enrolled in the study were provided with a dosimeter (QuietDose, Honeywell Inc, Morristown, New Jersey, USA) connected to microphones designed to fit inside of a variety of HPDs. The microphones were inserted into the volunteers' regular hearing protection, which included formable and premolded earplugs, custom-molded earplugs and earmuffs. The in-ear microphone measured noise levels inside the HPDs and delivered this information to the dosimeter, which was carried in the volunteer's pocket, or on a belt or hard hat. At the end of each work shift, the volunteer could download the noise exposure data into a computer located near the volunteer's usual workstation. At the time of download, the worker was able to view their daily noise dose for the work shift as a percentage of the OSHA Permissible Exposure Level, an 8-hour average of $90 \mathrm{dBA}$ using a $5 \mathrm{~dB}$ time-intensity exchange rate, a SLOW time constant, and an $80 \mathrm{~dB}$ threshold. Battery replacements and dosimeter calibration were provided by the research staff. Monthly summaries of exposures were also printed and sent to the study volunteers by research staff. Company management did not have access to individual participants' downloaded data but were provided summary results of aggregate noise exposures from different work areas on a regular basis.

\section{Surveillance audiometry and audiometric surveys}

On a yearly basis, all noise-exposed employees in the study facilities were required to undergo audiometric testing as part of the company Hearing Conservation Programme. Audiometric testing consisted of pure tone air conduction threshold audiometry at the test frequencies of $0.5,1,2,3,4,6$ and $8 \mathrm{kHz}$ conducted in a testing booth by a trained audiometric technician. At the time of their audiometric tests, subjects also completed a standardised screening survey. This survey addressed demographics, history of tinnitus and ear surgery, involvement in a range of noisy hobbies, use of firearms and previous or current employment in another noisy industry. Audiometric and survey results were linked to the study subjects by a data manager at Yale University in a confidential fashion through the use of a common scrambled identifier. For our analyses of hearing threshold levels and loss over time, we used the binaural average of the hearing threshold levels $(\mathrm{dB}$ $\mathrm{HL}$ ) at the noise sensitive frequencies of 3, 4 and $6 \mathrm{kHz}$.

\section{Matching controls}

We used the anonymous records of other workers in the facilities to identify control workers with similar noise exposures and demographic profiles. From among individuals at the 
same facilities who were enrolled in the hearing conservation programme, we searched for one control worker for each study volunteer. We made this selection based on matching for job title, age, gender, race, plant location and baseline hearing level.

\section{Estimated ambient noise exposure}

In addition to the noise exposure data from the daily monitoring devices, we also calculated an average ambient noise exposure for both the study volunteers and the controls. As previously described, ${ }^{1}$ we used records of noise monitoring from the company industrial hygiene database, and assigned average ambient noise exposures (outside of hearing protection) to each individual based on job title and job history.

\section{Data analysis}

In order to determine the outcome of hearing loss over the intervention period, we restricted our analysis to those volunteers who had downloaded at least five times, who had a baseline audiogram within a year prior to beginning the study, and who had received at least two audiograms at least 2 years apart between the baseline audiogram and any subsequent audiograms over the 5 -year period since joining the study.

Volunteer users were eliminated from further analysis if they had baseline average hearing levels at 3,4 and $6 \mathrm{kHz}$ of $>50 \mathrm{~dB}$ $\mathrm{HL}$ in order to eliminate subjects with substantial hearing loss at the start of the study. Volunteer users were also eliminated from further analysis if they showed an annual decline in hearing levels of $>10 \mathrm{~dB} \mathrm{HL}$ on average at 3,4 and $6 \mathrm{kHz}$, as loss of that magnitude is not consistent with the noise levels present at the participating location and suggested possible pathology or other non-exposure-related causation of hearing loss.

Out of 252 subjects who originally signed up for the study, 229 downloaded at least five times. After applying the inclusion criteria described previously, outcome data and control subjects were available for 110 individuals. Figure 1 (study cohort flow chart) shows how the cohort was assembled.

Data were analysed using SAS V.9.4 (SAS Institute).

We described the baseline demographic characteristics between volunteer dosimeter users and controls using means and proportions to identify differences between the intervention groups.

To test our primary hypothesis (that data from the in-ear dosimeters would increase risk awareness and self-efficacy and reduce NIHL risk among volunteer users), we calculated the slope of change of audiograms performed over the study period, using the binaural average of the noise sensitive frequencies of 3 , 4 and $6 \mathrm{kHz}$, as an annualised rate of change ( $\mathrm{dB} \mathrm{HL} /$ year). We compared these rates between volunteer users of the daily noise exposure monitoring device and the controls. We also examined the relationship between the number of downloads per year and hearing loss among the volunteer workers.

\section{RESULTS}

\section{Demographics and risk factors}

Table 1 shows the demographic characteristics of the 110 volunteer users and the 110 controls, as well as reported risk factors for hearing loss and the mean rate of loss ( $\mathrm{dB} / \mathrm{year})$ in the binaural average of 3, 4 and $6 \mathrm{kHz}$ over the study period. The group was predominantly male and Caucasian, with a similar mean age of about 44 years. Among the three study sites, ambient noise exposure estimates were higher in the fabrication plant compared with the smelters, and only in that plant were the average exposures in excess of the OSHA $85 \mathrm{dBA}$ Action Level. Certain risk factors for NIHL, including participation in another noisy job,

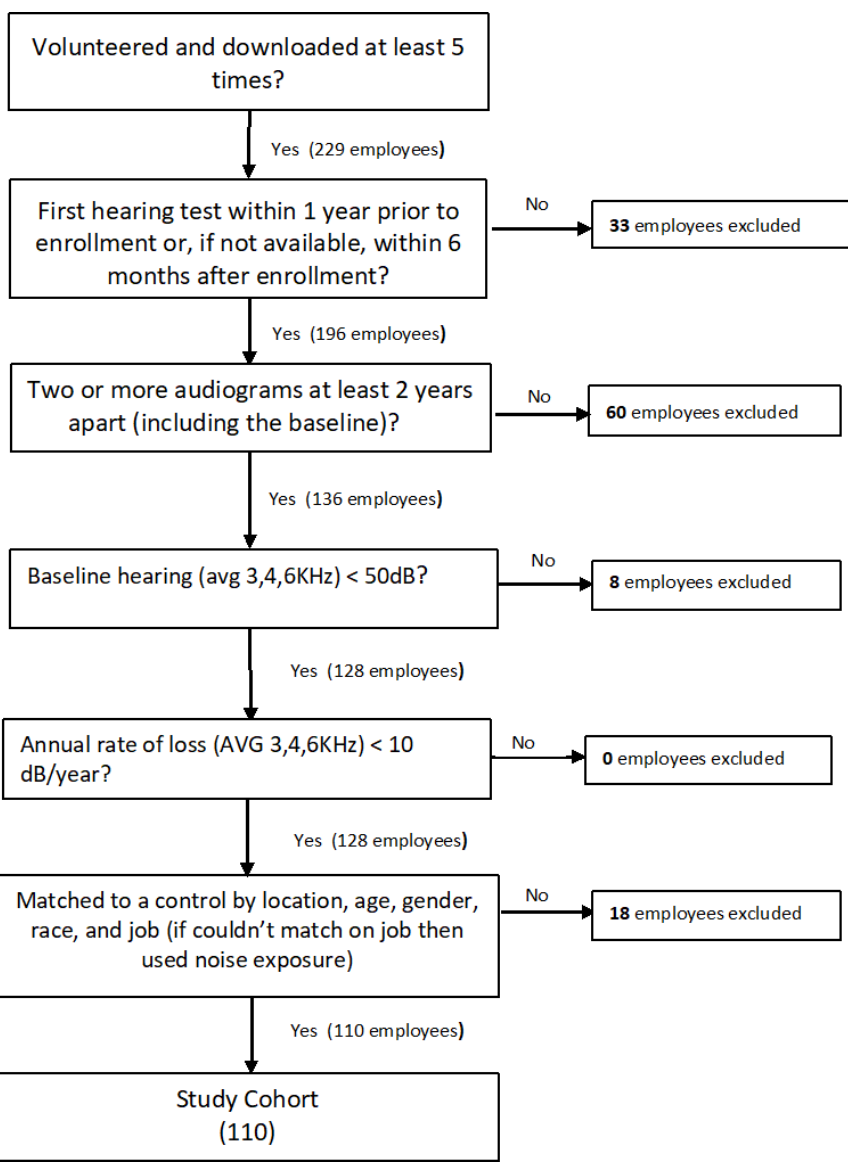

Figure 1 Construction of study cohort.

previous loud job and hunting and shooting were reported more frequently in the controls compared with the volunteers.

The overall rate of hearing loss did not differ significantly between volunteers and controls across all locations. In one of the three locations (Smelter Plant 1), however, the amount of hearing loss in the volunteers $(\mathrm{N}=11)$ was significantly greater than in the 11 controls from the same location.

\section{Noise exposures inside of hearing protection devices}

Table 2 shows the mean noise exposures (in $\mathrm{dBA}$ ) over the study duration estimated from company ambient noise monitoring records compared with noise levels measured by the volunteers inside of hearing protection using the daily noise exposure devices. The table shows that noise exposures were lower inside of hearing protection, indicating that volunteers were receiving protection from their hearing protective devices.

\section{Number of exposure downloads and hearing loss}

The 110 study volunteers recorded and downloaded their daily noise exposures an average of 149.9 times per year (SD 79.9, range 2.8-317.6) over the study observation period. While there was a slight trend toward less hearing loss with a greater number of downloads per year (figure 2), this trend did not achieve statistical significance ( $\mathrm{p}$ value for trend $=0.19$ ). However, when comparing volunteers who downloaded greater than 150 times per year versus those who downloaded less than 150 times per year (table 3), the high download group had a lower rate of hearing loss $(0.33 \mathrm{~dB} \mathrm{HL} / \mathrm{year}$ vs $0.98, \mathrm{p}=0.017)$ compared with the low download group, despite also having slightly higher measured in-ear noise levels (78.6 vs $74.3 \mathrm{dBA}, \mathrm{p}=0.001)$. This 


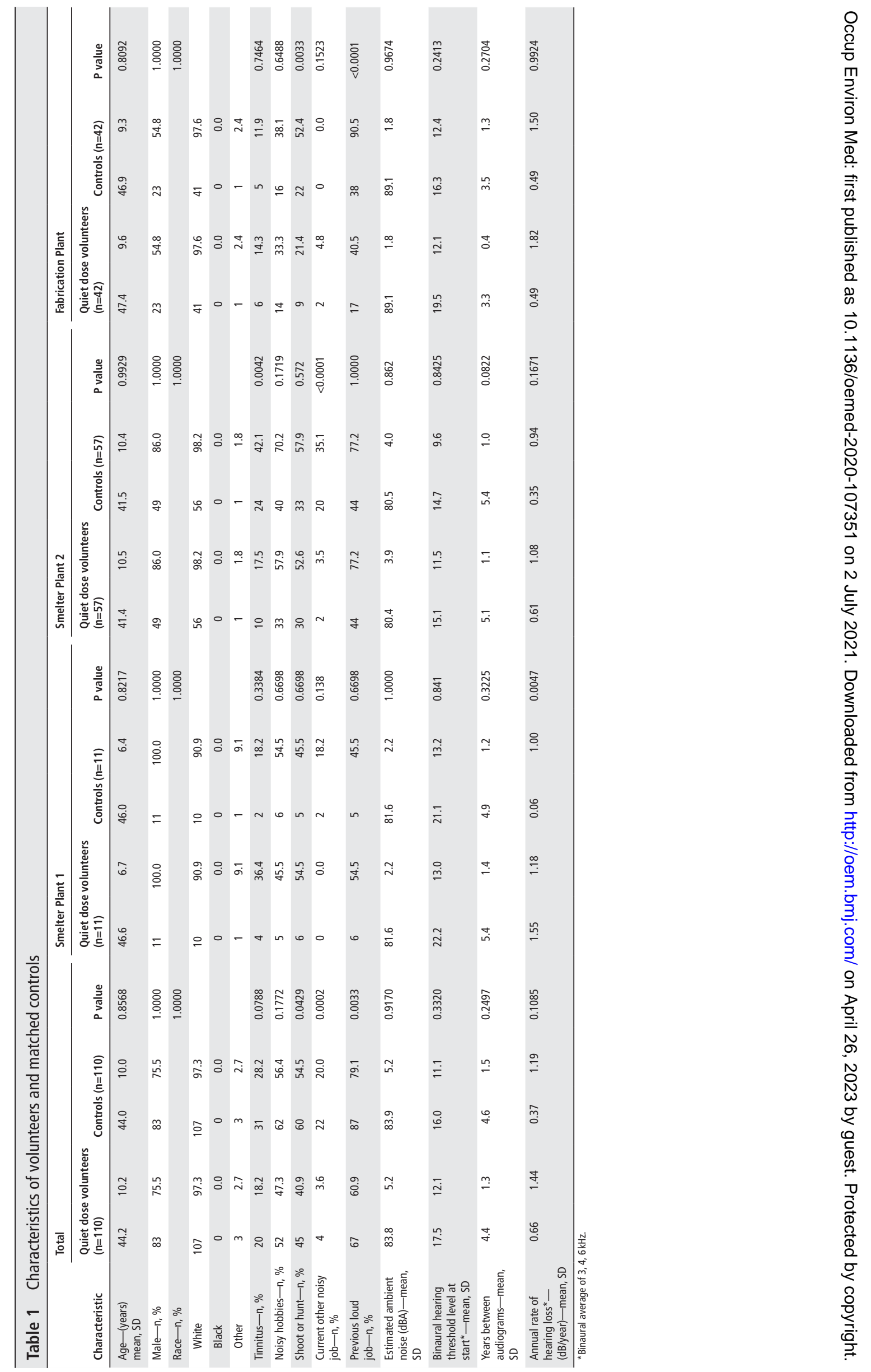


Table 2 Estimated ambient noise exposures (dBA) versus measured noise inside of hearing protection (dBA): study volunteers, by location

\begin{tabular}{llllll}
\hline & \multicolumn{2}{l}{ Estimated ambient noise $(\mathrm{dBA})$} & & \multicolumn{2}{l}{ In-ear noise dosimetry (dBA) } \\
\cline { 2 - 3 } \cline { 5 - 6 } Location & Mean & SD & & Mean & SD \\
\hline Smelter 1 & 81.6 & 2.2 & 72.4 & 4.2 \\
Smelter 2 & 80.4 & 3.9 & 74.0 & 6.4 \\
Fabrication & 89.1 & 1.8 & 80.9 & 6.3 \\
\hline
\end{tabular}

difference remained significant after adjusting for the covariates listed in table $3(\mathrm{p}=0.0127)$.

\section{DISCUSSION}

The results of this study suggest that voluntary daily monitoring of noise exposure by noise-exposed workers is feasible, and resulted in documentation of noise levels inside of the hearing protectors that were lower than ambient noise measurements, and less than the action level for hearing conservation programmes under the OSHA Action Level (85 dBA TWA). While many volunteers downloaded only a few times, some individuals performed hundreds or even thousands of downloads over the study period. Measured levels of noise inside of the hearing protectors were lower than ambient noise estimates for job titles from the company industrial hygiene database. While overall the study volunteers did not exhibit lower rates of hearing loss compared with a control group of workers from the same company with similar demographics, we found that workers who downloaded more than 150 times per year had less hearing loss than those who downloaded less frequently.

The finding of lower noise levels inside of hearing protection compared with estimated ambient exposures was consistent with an earlier report that use of the devices appeared to reduce noise exposure in volunteers. ${ }^{16}$ There are a number of possible mechanisms by which daily noise exposure monitoring could reduce noise exposure and consequently hearing loss risk. Workers who receive daily feedback from a monitoring device about their noise exposure could become motivated to make changes in the way that they use HPDs. These changes could include alterations in either the attenuation achieved when HPDs are worn through modification of the fit of the HPDs, or modifications to the timing and duration of their use. Either or both of these alterations could result in improved overall performance of the HPDs. Such feedback could also lead workers to identify sources of excessive noise and either avoid or bring those to the attention of management to the noise, which could ultimately result in reduced noise exposure through implementation of engineering or administrative noise controls. While study volunteers anecdotally reported both of these events, we did not systematically capture such effects in our data collection. Future studies should explore the relationship between daily noise monitoring and the effect on HPD use and noise avoidance behaviour.

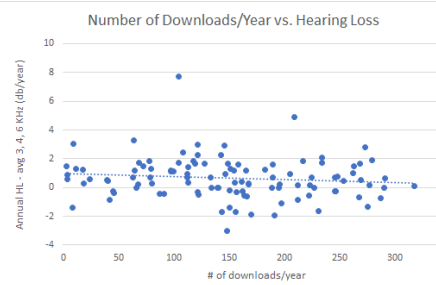

Figure 2 Number of downloads per year and rate of hearing loss ( $\mathrm{dB} \mathrm{HL} /$ year) for study volunteers.
Table 3 Comparison of hearing loss and other variables between volunteers according to frequency of downloading

\begin{tabular}{|c|c|c|c|c|c|}
\hline \multirow{3}{*}{$\begin{array}{l}\text { Characteristic } \\
\text { Age-mean, SD }\end{array}$} & \multicolumn{5}{|c|}{ Downloads per year } \\
\hline & \multicolumn{2}{|c|}{$<150 /$ year $(n=55)$} & \multicolumn{2}{|c|}{$>150 /$ year $(n=55)$} & \multirow{2}{*}{$\begin{array}{l}P \text { value } \\
0.0813\end{array}$} \\
\hline & 42.5 & 9.9 & 45.9 & 10.3 & \\
\hline Male-n, \% & 41 & 74.6 & 42 & 76.4 & 0.8247 \\
\hline Race-n, \% & & & & & 0.5583 \\
\hline White & 54 & 98.2 & 53 & 96.4 & \\
\hline Tinnitus—n, \% & 11 & 20.0 & 9 & 16.4 & 0.6210 \\
\hline Noisy hobbies-n, \% & 30 & 54.6 & 22 & 40.0 & 0.1266 \\
\hline Shoot or hunt-n, \% & 22 & 40.0 & 23 & 41.8 & 0.8462 \\
\hline $\begin{array}{l}\text { Current other noisy } \\
\text { job-n, } \%\end{array}$ & 3 & 5.5 & 1 & 1.8 & 0.3083 \\
\hline Previous loud job-n, \% & 38 & 69.1 & 29 & 52.7 & 0.0786 \\
\hline $\begin{array}{l}\text { Ambient noise (dBA)—- } \\
\text { mean, SD }\end{array}$ & 83.3 & 5.0 & 84.4 & 5.3 & 0.2713 \\
\hline $\begin{array}{l}\text { In-ear noise dosimetry } \\
\text { level }(\mathrm{dBA}) \text {-mean, SD }\end{array}$ & 74.3 & 7.0 & 78.6 & 6.5 & 0.0014 \\
\hline $\begin{array}{l}\text { Hearing at start }{ }^{*}- \\
\text { mean, SD }\end{array}$ & 16.3 & 11.8 & 18.7 & 12.3 & 0.2915 \\
\hline $\begin{array}{l}\text { Years between } \\
\text { audiograms—-mean, SD }\end{array}$ & 4.6 & 1.3 & 4.3 & 1.2 & 0.2809 \\
\hline $\begin{array}{l}\text { Annual rate of hearing } \\
\text { loss*_-dB/year-mean, } \\
\text { SD }\end{array}$ & 0.98 & 1.55 & 0.34 & 1.25 & 0.0171 \\
\hline
\end{tabular}

${ }^{*}$ Binaural average of $3,4,6 \mathrm{kHz}$.

There are a number of possible explanations for the lack of an observed overall benefit on hearing loss rates when volunteers were compared with controls. The difference between the two groups was less than $0.5 \mathrm{~dB} \mathrm{HL}$ per year, and of doubtful clinical significance. Since all of the study locations had active hearing conservation programmes, the ongoing efforts to reduce noise exposures through hearing protection and other measures could have reduced the incidence of NIHL among all noiseexposed workers in those locations to a risk level low enough to obscure any benefit from the intervention. The low rate of hearing loss over the study period was suggested by the fact that the calculated slope of change for some workers was less than 0 $\mathrm{dB} /$ year, probably due to testing variability between subsequent tests that could be due to changes in background noise levels, learning effect or other factors. We relied on company audiometric records, and therefore were unable to determine or adjust for fluctuations in background noise levels, equipment calibration, testing instruction or other test variables. Given that the overall risk of NIHL was low, the sample size could have been too small to demonstrate a significant difference. Another possibility was that the mean follow-up period of 4.4 years may have not been long enough to capture the gradual effect of NIHL. The fact that the average age of the volunteers and controls was over 40 implies that many workers had already had years of noise exposure and this pre-existing noise exposure could have limited the rate of further loss. While it is possible that unmeasured confounders such as non-occupational noise exposures could have accounted for the lack of demonstrated benefit of the noise monitoring device, the control individuals actually reported higher rates of hunting, shooting, other noisy job and noisy hobbies.

At the same time, we observed an overall trend toward less hearing loss with more frequent downloading of daily exposure data by the volunteers, and significantly less hearing loss in the volunteers who downloaded on a more frequent basis. This 
suggests that compliance with daily downloading protocols could be a key determinant of the effectiveness of the intervention.

In a previous study at another location, we have reported that mandatory use of daily noise exposure monitoring by noiseexposed workers was associated with reduced risk of hearing loss. ${ }^{15} \mathrm{~A}$ possible explanation for the finding that volunteers in the current study did not demonstrate less hearing loss than the controls could be that voluntary use was less consistent than the mandatory usage previously reported. While the volunteers downloaded an average of 150 times per year, the range of usage was wide, and some volunteers used the monitoring device much less than that. In a previous qualitative study of usability of the devices, volunteers reported a number of barriers to daily use, including the wires connecting the microphones to the dosimeter, and the inconvenience of downloading at a workstation. ${ }^{17}$ It is therefore likely that barriers remain to voluntary monitoring, even with the incentives provided by the study. This is supported by the number of volunteers who dropped out of the study or failed to download very often. If the technology for self-monitoring of noise exposure can continue to evolve, such as development of wireless devices that do not require a manual download, it is possible that such barriers will diminish, making self-monitoring even more feasible and possibly more effective in the future.

We believe that this study supports the feasibility of worksite noise exposure monitoring that provides regular feedback to workers regarding their occupational noise exposure. Hearing conservation programmes should consider means of providing more frequent and personalised monitoring and feedback about exposures to those working in areas with potentially damaging noise exposure. This approach, combined with a robust noise control effort, has the potential to further reduce the incidence of NIHL among noise-exposed workers.

Acknowledgements The authors thank Vickie Ramirez for assistance in the preparation of this manuscript. We also thank the study site coordinators, and above all the study volunteers.

Contributors Conceptualisation of the work: CD-E, RN, PR. Methodology: LFC, DG, RN, PR. Analysis of data: DG. Writing original draft: RN, PR. Writing-reviewing and editing: LFC, CD-E, DG.

Funding This study was supported by funding from the National Institute of Occupational Safety and Health (CDC/NIOSH) Grant 1 R01 OH008641-01A2 'Daily Exposure Monitoring Intervention to Prevent Hearing Loss'.

Competing interests CD-E was an employee of the company where the study took place. PMR, DG and LFC had received funding from the study company through an agreement with Yale University for occupational health consultation not directly related to this study.
Patient consent for publication Not required.

Ethics approval Study protocols were reviewed and approved by the Yale School of Medicine Human Investigation Committee (\#0509000588).

Provenance and peer review Not commissioned; externally peer reviewed.

Data availability statement Data are available upon reasonable request. A data set for variables related to the study volunteers as well as the analytic protocols is available from the lead author for the purpose of verification of findings. Requests can be sent to peterr7@uw.edu.

\section{ORCID iD}

Richard Neitzel http://orcid.org/0000-0001-5500-2589

\section{REFERENCES}

1 Cantley LF, Galusha D, Cullen MR, et al. Association between ambient noise exposure, hearing acuity, and risk of acute occupational injury. Scand I Work Environ Health 2015; $41: 75-83$.

2 Arlinger S. Negative consequences of uncorrected hearing loss-a review. Int J Audiol 2003;42:17-20.

3 Lusk SL. Noise exposures. Effects on hearing and prevention of noise induced hearing loss. Aaohn J 1997; 45:397-408.

4 Masterson EA, Tak S, Themann CL, et al. Prevalence of hearing loss in the United States by industry. Am J Ind Med 2013;56:670-81.

5 Basner M, Babisch W, Davis A, et al. Auditory and non-auditory effects of noise on health. Lancet 2014:383:1325-32.

6 Cantley LF, Galusha D, Cullen MR, et al. Does tinnitus, hearing asymmetry, or hearing loss predispose to occupational injury risk? Int J Audiol 2015;54 Suppl 1:S30-6.

7 Verbeek JH, Kateman E, Morata TC, et al. Interventions to prevent occupational noise-induced hearing loss: a Cochrane systematic review. Int J Audiol 2014;53 Suppl 2:S84-96.

8 Neitzel R, Somers S, Seixas N. Variability of real-world hearing protector attenuation measurements. Ann Occup Hyg 2006;50:679-91.

9 Reddy RK, Welch D, Thorne $\mathrm{P}$, et al. Hearing protection use in manufacturing workers: a qualitative study. Noise Health 2012;14:202.

10 El Dib RP, Verbeek J, Atallah AN, et al. Interventions to promote the wearing of hearing protection. Cochrane Database Syst Rev 2006:CD005234.

11 Laplante-Lévesque A, Brännström KJ, Ingo E, et al. Stages of change in adults who have failed an online hearing screening. Ear Hear 2015;36:92-101.

12 Manchaiah V, Rönnberg J, Andersson G, et al. Stages of change profiles among adults experiencing hearing difficulties who have not taken any action: a cross-sectional study. PLoS One 2015;10:e0129107.

13 Prochaska JO, Velicer WF. The transtheoretical model of health behavior change. Am J Health Promot 1997; 12:38-48.

14 Seixas NS, Neitzel R, Stover B, et al. A multi-component intervention to promote hearing protector use among construction workers. Int J Audiol 2011;50 Suppl 1:S46-56.

15 Rabinowitz PM, Galusha D, Kirsche SR, et al. Effect of daily noise exposure monitoring on annual rates of hearing loss in industrial workers. Occup Environ Med 2011;68:414-8.

16 McTague MF, Galusha D, Dixon-Ernst C, et al. Impact of daily noise exposure monitoring on occupational noise exposures in manufacturing workers. Int I Audiol 2013;52 Suppl 1:S3-8.

17 Williams SC, Rabinowitz PM. Usability of a daily noise exposure monitoring device for industrial workers. Ann Occup Hyg 2012;56:925-33. 